\title{
Memantine and Functional Communication in Alzheimer's Disease: Results of a 12-Week, International, Randomized Clinical Trial
}

\author{
Judith Saxton ${ }^{\mathrm{a}, *}$, Robert K. Hofbauer ${ }^{\mathrm{b}}$, Michael Woodward ${ }^{\mathrm{c}}$, Nigel L. Gilchrist ${ }^{\mathrm{d}}$, Felix Potocnik ${ }^{\mathrm{e}}$, \\ Hai-An Hsu ${ }^{\mathrm{b}}$, Michael L. Miller ${ }^{\mathrm{f}}$, Vojislav Pejović ${ }^{\mathrm{f}}$, Stephen M. Graham ${ }^{\mathrm{b}}$ and James L. Perhach ${ }^{\mathrm{b}}$ \\ a Alzheimer's Disease Research Center, Department of Neurology, University of Pittsburgh, Pittsburgh, PA, USA \\ ${ }^{\mathrm{b}}$ Forest Research Institute, Jersey City, NJ, USA \\ ${ }^{\mathrm{c}}$ Austin Health, Medical \& Cognitive Research Unit, Heidelberg West, VIC, Australia \\ ${ }^{\mathrm{d}}$ CGM Research Trust, The Princess Margaret Hospital, Christchurch, New Zealand \\ ${ }^{\mathrm{e}}$ Flexivest 14 Research Centre, Bellville, Western Cape, South Africa \\ ${ }^{\mathrm{f}}$ Prescott Medical Communications Group, Chicago, IL, USA
}

Handling Associate Editor: Montse Alegret

Accepted 19 August 2011

\begin{abstract}
Post hoc analyses suggest that memantine treatment may provide communication-related benefits in patients with Alzheimer's disease (AD). In this 12-week, international, randomized, double-blind, placebo-controlled trial of memantine (10 mg bid), the functional communication abilities of patients with AD (MMSE range: 10-19) were assessed using the Functional Linguistic Communication Inventory (FLCI; primary measure). Two combined subscales (Social Communication and Communication of Basic Needs) from the American Speech-Language-Hearing Association Functional Assessment of Communication Skills for Adults (ASHA FACS; secondary measure) were administered to caregivers. Treatment-emergent adverse events were also recorded. After 12 weeks, memantine-treated patients $(n=133)$ demonstrated a non-significant improvement on the FLCI (placebo: -0.6 ; memantine: $0.7 ; p=0.070$, LOCF) and a significant improvement on the ASHA FACS (placebo: -5.3; memantine: $0.5 ; p=0.022)$, compared with placebo-treated patients $(n=124)$. Memantine had a low incidence of adverse events. In patients with moderate $\mathrm{AD}$, memantine treatment improved functional communication, as recognized by caregivers.
\end{abstract}

Keywords: Alzheimer's disease, American Speech-Language-Hearing Association Functional Assessment of Communication Skills for Adults (ASHA FACS), communication, drug therapy, Functional Linguistic Communication Inventory (FLCI), language, memantine, randomized controlled trial

\footnotetext{
*Correspondence to: Judith Saxton, PhD, Clinical Core Director, Alzheimer's Disease Research Center, University of Pittsburgh, PA, 200 Lothrop Street, Pittsburgh, PA 15213-2536, USA. Tel.: +(412) 692 2885; Fax: +(412) 692 4031; E-mail: saxtonja@upmc.edu.
}

\section{INTRODUCTION}

Communication is central to all human activities and interactions. Individuals with Alzheimer's disease (AD) lose particular communication abilities during the mild, moderate, and severe stages of the disease [1]. Communication deficits in the 
moderate stage of AD include problems with wordfinding, confrontation naming, and verbal fluency [1-4]. In addition, increasing impairment is observed in "functional communication," which the American Speech-Language-Hearing Association (ASHA) defines as "the ability to receive or to convey a message, regardless of the mode, to communicate effectively and independently in a given environment" $[5,6]$. The inability of patients to communicate effectively may lead to adverse behaviors such as agitation and aggression, which can result in significant stress and burden for caregivers [7, 8].

In AD clinical trials, outcome measures typically include instruments that measure cognition, functional abilities, global status, and behavior; however, instruments that measure specific deficits in language and functional communication have rarely been used. Nevertheless, post hoc analyses suggest that the therapies approved for the treatment of AD may improve patients' functional communication across the severity spectrum of the disease. For example, a retrospective, pooled analysis of four 26-week trials of rivastigmine, a cholinesterase inhibitor (ChEI), demonstrated that rivastigmine-treated patients with mild to moderate AD improved significantly on the language domain of the AD Assessment Scale-cognitive subscale (ADAScog), compared with their placebo-treated counterparts [9]. Similarly, in patients with moderate to severe $\mathrm{AD}$, post-hoc analyses suggest that treatment with memantine, an uncompetitive antagonist of N-methyl$\mathrm{D}$-aspartate (NMDA) receptors, is associated with significantly better performance than placebo on the language domain of the Severe Impairment Battery (SIB) $[10,11]$, as well as on individual items involving language and communication from the SIB, the AD Cooperative Study-Activities of Daily Living scale (ADCS-ADL; 19-item version), and the Behavioral Rating Scale for Geriatric Patients (BGP) [12]. Furthermore, in an international, randomized, double-blind, placebo-controlled trial in patients with moderate to severe $\mathrm{AD}$, an extended-release memantine formulation was associated with significant benefits on the prospectively defined measure of verbal fluency, compared with placebo [13]. Also, in studies of individuals suffering from post-stroke aphasia, ChEIs [14] as well as memantine [15] have been associated with improvements in communication abilities. Finally, a recent open-label trial in patients with moderate to severe $\mathrm{AD}$ demonstrated that once-daily memantine treatment was associated with significant improvement in functional communication after 12 weeks [16].
The purpose of this randomized, double-blind, placebo-controlled trial was twofold: 1) to prospectively examine the effects of memantine treatment on functional communication in patients with moderate $\mathrm{AD}$, and 2) to evaluate the utility of the Functional Linguistic Communication Inventory (FLCI) [17] and the Functional Assessment of Communication Skills for Adults (ASHA FACS) scale [18], two measures of functional communication, as assessment tools in a randomized clinical trial in AD.

\section{MATERIALS AND METHODS}

\section{Trial design}

This 12-week trial (MEM-MD-71; NCT00469456) began recruiting in April 2007, with the first patient visit on May 24, 2007. The last patient visit occurred on November 4, 2008. Participants were recruited from 25 centers: 14 in Australia, 8 in South Africa, and 3 in New Zealand. The 265 participants (male and female, $\geq 50$ years of age) were native English speakers who had a diagnosis of probable AD, according to criteria set forth by the National Institute of Neurological and Communicative Disorders and Stroke-Alzheimer's Disease and Related Disorders Association (NINCDS-ADRDA), a Mini-Mental State Examination (MMSE) [19] score of 10-19 at both Screening (Week 2) and Baseline (Week 0), and computed tomography (CT) or magnetic resonance imaging (MRI) results within the past 12 months consistent with this diagnosis. Concurrent treatment with a ChEI was permitted but not required; in those receiving ChEI therapy, a stable dose of a single ChEI was required for at least 3 months prior to study entry and throughout the study. Subjects were required to pass a physical examination, clinical laboratory evaluation, and electrocardiogram (ECG) at Screening (Visit 1); they also had to be ambulatory or ambulatory-aided, with vision and hearing capabilities sufficient for compliance with testing procedures. Females were required to be surgically sterile or postmenopausal for at least 2 years. All subjects were required to have a knowledgeable and reliable caregiver who spoke English and could accompany the patient to all visits. Caregivers were required to spend sufficient time with the patients that they could accurately describe changes in the patients' cognitive, functional, and language abilities.

Exclusion criteria included clinically significant and active pulmonary, gastrointestinal, renal, hepatic, endocrine, or cardiovascular system disease 
or cancer; evidence of psychiatric or neurologic disorders other than probable $\mathrm{AD}$; dementia complicated by other organic brain disease or predominant delusions; clinically significant deficiency in vitamin $\mathrm{B}_{12}$ or folate; Hachinski Ischemic Score [20] $>4$ at Screening; hypertension (sitting systolic blood pressure $[\mathrm{SBP}]>180 \mathrm{~mm} \mathrm{Hg}$; diastolic blood pressure $[\mathrm{DBP}]>100 \mathrm{~mm} \mathrm{Hg}$ ); hypotension (sitting $\mathrm{SBP}<90 \mathrm{~mm} \mathrm{Hg}$; DBP $<50 \mathrm{~mm} \mathrm{Hg}$ ); history of alcoholism or drug abuse within 5 years prior to Screening; evidence of impaired kidney function or severe renal impairment; prior treatment with memantine, participation in a memantine clinical trial, or hypersensitivity to amantadine or rimantadine; participation in an investigational drug study or treatment with an investigational drug within 30 days (or 5 half-lives, whichever is longer) of Screening; likely institutionalization during the trial; any other condition that, in the opinion of the investigator, would make the patient or caregiver unsuitable for the study. Patients taking unapproved concomitant medications at any point throughout the trial were required to be discontinued from the trial. Written informed consent was provided by the patient (if possible) or legally authorized representative, as well as the patient's caregiver. This trial was approved by Institutional Review Boards (IRBs) at each participating site in Australia, and by the Central IRB in New Zealand and South Africa.

\section{Interventions}

This study was a randomized, double-blind, placebo-controlled trial in which participants were required to complete up to 2 weeks of open-label, placebo-only treatment immediately prior to Baseline, after which they were randomly assigned to treatment with either memantine $(10 \mathrm{mg}$ bid) or placebo (1:1 ratio). The Statistical Programming department at Forest Research Institute (FRI) generated a list of patient randomization codes, identifying each patient by randomization number and treatment assignment. Randomization numbers were assigned sequentially at each study site as each patient entered the study. A hard copy of the randomization list was maintained and stored in a secure area by Drug Safety Surveillance at FRI. Medication corresponding to the randomization numbers, which included an identifying tear-off panel for the patient's medication accountability form, was provided to each study site.

Patients assigned to double-blind memantine treatment were titrated in weekly increments of $5 \mathrm{mg}$, reaching the maximum, target dose of $10 \mathrm{mg}$ bid at the beginning of Week 4. Placebo-treated patients were given tablets identical in number and appearance to those containing memantine. All actively treated patients were required to reach the target dose, and dosage modifications were not permitted for patients experiencing dose-limiting adverse events. After completion of the trial, all patients were provided up to 6 months of open-label memantine treatment.

\section{Outcome measures}

Efficacy

The primary outcome measure was the mean change from Baseline on the FLCI [17], a direct, performance-based tool designed to assess functional communication in patients with moderate to severe $\mathrm{AD}$. The instrument, which takes approximately 30 minutes to complete, evaluates 10 areas: greeting and naming, answering questions, writing, sign comprehension and object-to-picture matching, word reading and comprehension, reminiscing, following commands, pantomime, gesture, and conversation. Approximately half of the items included in the FLCI were derived from a research battery used in a fiveyear longitudinal study of 91 patients with $\mathrm{AD}$; the other items were added later to further assess the effects of the disease on functional communication [17]. The combined set of items was administered to a standardization sample of 40 patients with $\mathrm{AD}$. The FLCI has demonstrated the ability to differentiate between patients in moderately severe (mean MMSE 6.8; bladder-incontinent but not bedridden), severe (mean MMSE 1.5; bowel- and bladder-incontinent but not bedridden), and very severe (mean MMSE 0.0 ; bowel/bladder-incontinent and bedridden) stages of $\mathrm{AD}[2,17]$. The measure demonstrates high test-retest reliability and has been validated [17] against the Arizona Battery for Communication Disorders of Dementia (ABCD), a standardized test of linguistic communicative function in patients with AD [21]. The score range for the FLCI is $0-87$, with higher scores indicating higher levels of functioning [17]. FLCI assessments were completed at Screening, Baseline, and Weeks 4, 8, and 12 (Endpoint).

The secondary outcome measure was the mean change from Baseline on two combined subscales from the ASHA FACS: Social Communication (SC) and Communication of Basic Needs (CBN) [18, 22]. The ASHA FACS, used by speech-language pathologists in the United States and other countries [18, 22], is a caregiver rating scale that was designed to assess functional 
communication skills in a wide variety of patients with disorders of speech, language, and cognitive communication. It was initially validated for use in dementia and shown to correlate significantly with MMSE scores in a sample of 15 patients (coefficient for the total score: $0.716 ; p<0.01$ ) [22]; subsequently, in a sample of 108 Brazilian subjects with mild $\operatorname{AD}(n=32)$, moderate $\mathrm{AD}(n=25)$, and elderly control subjects without dementia $(n=51)$, the Portuguese version of the scale demonstrated good sensitivity $(75.0 \%)$ and specificity $(82.4 \%)$, with high internal consistency (Cronbach $\alpha=0.955$ ), test-retest reliability (interclass $r=0.995$; $p<0.001$ ), inter-examiner reproducibility (interclass $r=0.998 ; p<0.001)$ and criterion validity when correlated with ADAS-cog $(r=-0.69 ; p<0.001)$ [5]. The total ASHA FACS takes approximately $20 \mathrm{~min}$ to complete and evaluates 4 areas: social communication, communication of basic needs, daily planning, and reading/writing/number concepts. The score range for the total ASHA FACS is 0-301, with higher scores indicating higher levels of functioning; the combined score range for the two ASHA FACS subscales ( $\mathrm{SC}$ and $\mathrm{CBN}$ ) utilized as the secondary outcome measure in this study is $0-196$, with higher scores indicating higher levels of functioning. The ASHA FACS was administered at Screening, Baseline, and Weeks 4, 8, and 12 (Endpoint). The ASHA FACS total score was utilized as an additional outcome measure.

Another additional outcome measure was the Clinical Global Impression of Change (CGI-C) [23], an assessment performed by the clinician through direct observation of the patient, with separate input from the caregiver. CGI-C ratings range from 1 (very much improved) to 7 (very much worse); a score of 4 indicates no clinically relevant change. The CGI-C was assessed at Weeks 4, 8, and 12 (Endpoint). Additional protocol-specified CGI measures were used to assess global change in social interaction and communication.

Two additional measures, the Oral Production Test (OPT) and the Caregiver Perceived Burden Questionnaire (CPBQ) were administered at Baseline and Week 12 (Endpoint). The OPT, a component of the Neuropsychological Assessment Battery [24], evaluates a patient's speech output and fluency while describing a picture of a family scene. The CPBQ [8], an exploratory measure that was not analyzed as a part of this report, is designed to assess the quality of a patient's speech, social interaction and awareness, and ability to manage daily activities as it affects the caregiver.

\section{Safety and tolerability}

Clinical laboratory determinations, a standard 12-lead electrocardiogram (ECG), and a physical examination were performed at Screening; vital signs, including weight, were performed at Screening and all study visits. Treatment-emergent adverse events (TEAEs) and serious adverse events (SAEs) were recorded at all post-Screening study visits. The number (percentage) of patients with potentially clinically significant post-Baseline values of vital sign parameters were tabulated by treatment group.

\section{Data analysis}

The Safety Population consisted of all randomized patients who took at least one dose of double-blind study medication. The Intent-to-Treat (ITT) Population consisted of all patients in the Safety Population who had at least one post-Baseline assessment of the primary efficacy parameter, the FLCI. Primary and secondary outcomes were analyzed by comparing least squares mean differences (LSMD) at Endpoint in the two study groups (ITT population), using the last observation carried forward (LOCF) approach for imputation of missing data. Supporting analyses were based on the observed cases (OC) approach, as well as on a mixed-effects model with repeated measures (MMRM; FLCI only). A two-way ANCOVA model was used for these analyses, with treatment group and study center as factors and Baseline value as covariate. CGI data were analyzed using a Cochran-MantelHaenszel test, controlling for study center and using modified ridit scores. All tests were two-sided, with $\alpha=0.05$.

\section{Sample size}

Since clinical data regarding an expected treatment effect of the FLCI were not available, and since the FLCI and SIB are similar in score range and type of assessment, the sample size was estimated based on pooled data from the Severe Impairment Battery (SIB) from 3 previously completed memantine studies (memantine: 249; placebo: 277) [25-27]. In this pooled sample, a mean \pm SD treatment difference of $2.91 \pm 7.81$ points in favor of memantine was observed at Week 12. Using this estimate, a minimum of 125 patients per group were determined to be required to detect a similar treatment effect with a power greater than $80 \%$, using a two-sided, two sample $t$-test at a significance level of 0.05 . 


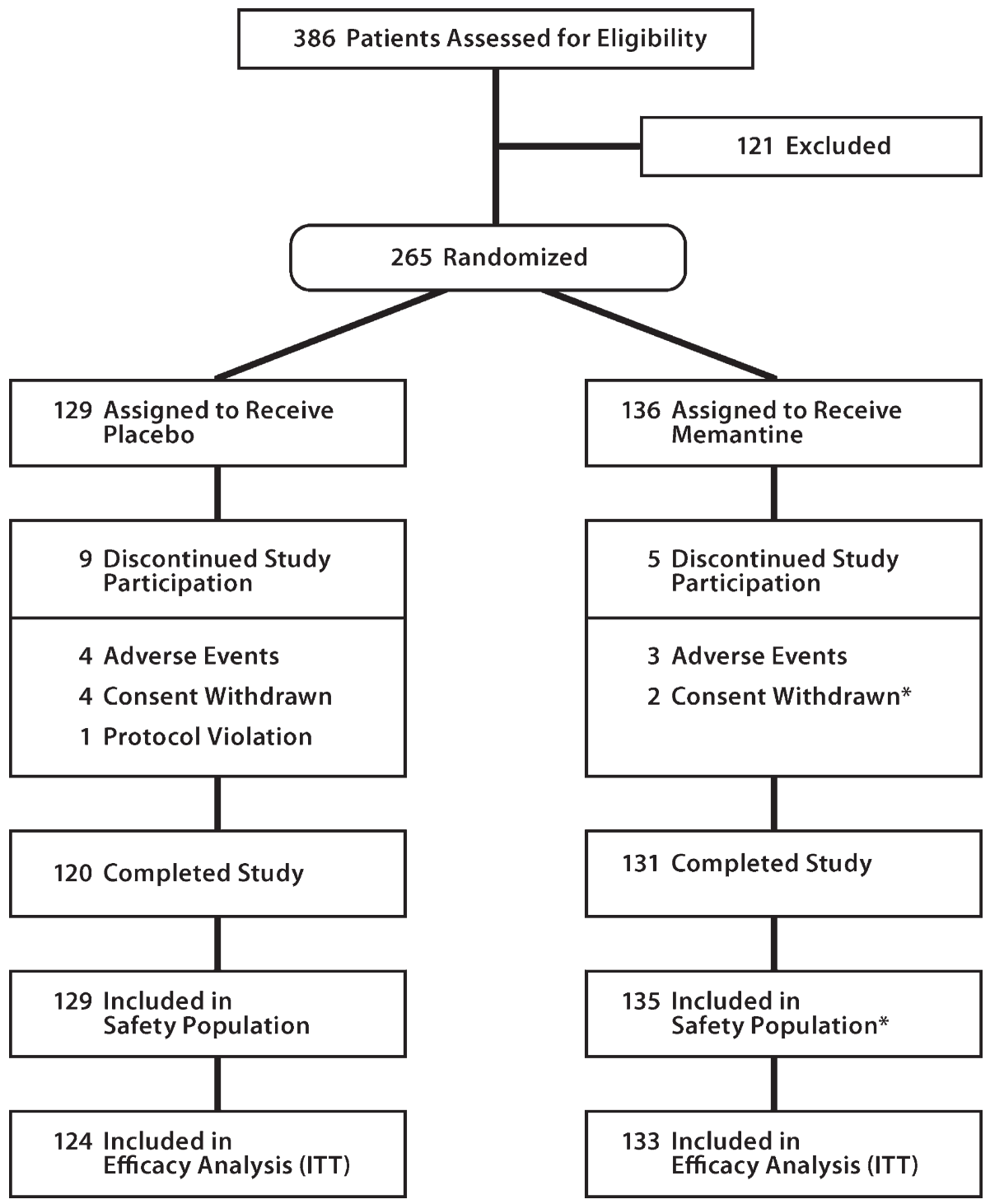

Fig. 1. Study flow.

One patient in the memantine group discontinued the trial without receiving study medication (withdrew consent) and was not included in the Safety Population. ITT indicates Intent-to-Treat Population.

\section{RESULTS}

\section{Patients}

The trial flow diagram is presented in Fig. 1. A total of 129 and 136 patients were randomized to receive placebo and memantine, respectively, with 120 (93.0\%) and $131(96.3 \%)$ completing the trial; four participants in the placebo group and three in the memantine group discontinued due to AEs (Fig. 1). The demographic and clinical characteristics of the study population are shown in Table 1 . Placebo and memantine groups were well matched at Baseline. No clinically significant differences were noted.

Efficacy

\section{Primary outcome measure: FLCI}

Memantine treatment showed a non-significant improvement over placebo on the FLCI at Week 12 (LOCF: $p=0.070$; OC: $p=0.184$ ), with statistically significant differences in favor of memantine at Week 4 (LOCF/OC: $p=0.028$ ) and Week 8 (LOCF: $p=$ 0.015; OC: $p=0.023$ ) (Fig. 2A, Table 2). The MMRM 
Table 1

Summary of baseline patient characteristics (safety population)

\begin{tabular}{lcc}
\hline Parameter & $\begin{array}{c}\text { Placebo } \\
(n=129)\end{array}$ & $\begin{array}{c}\text { Memantine } \\
(n=135)\end{array}$ \\
\hline Age, years* & $75.1 \pm 8.7[53-92]$ & $74.8 \pm 8.1[52-94]$ \\
Women, $n(\%)$ & $74(57.4)$ & $80(59.3)$ \\
White, $n(\%)$ & $118(91.5)$ & $122(90.4)$ \\
Weight, kg* & $69.2 \pm 13.9[39.9-107.3]$ & $68.1 \pm 13.2[35.0-114.0]$ \\
Education, years* & $11.3 \pm 3.0[0-18]$ & $11.7 \pm 2.9[5-24]$ \\
MMSE score* & $16.0 \pm 2.5[10-20]^{\dagger}$ & $15.7 \pm 2.7[10-19]$ \\
Concomitant ChEI treatment, $n(\%)$ & & \\
Donepezil & $39(30.2)$ & $44(32.6)$ \\
Galantamine & $27(20.9)$ & $27(20.0)$ \\
Rivastigmine & $2(1.6)$ & $1(0.7)$ \\
\hline
\end{tabular}

${ }^{*}$ Mean \pm standard deviation [range].

${ }^{\dagger}$ One randomized patient with an MMSE score of 19 at Screening scored a 20 at Baseline. ChEI indicates cholinesterase inhibitor; MMSE indicates Mini-Mental State Examination.

analysis indicated a statistically superior performance of the memantine group at Week $8(p=0.022)$ and across the entire trial (LSMD [95\% CI]: 1.3 [0.2, 2.40]; $p=0.021$ )

\section{Secondary outcome measure: ASHA FACS subscales}

Caregivers of memantine-treated patients reported significantly better patient communication, compared with caregivers of placebo-treated patients, on the combined ASHA FACS subscales of SC and CBN at Weeks 12 (LOCF: $p=0.022$; OC: $p=0.033$ ) and 8 (LOCF: $p=0.008$; OC: $p=0.006$ ) (Fig. 2B, Table 2).

\section{Additional outcome measures: CGI-C, ASHA FACS total score, and OPT}

Memantine treatment was associated with a greater proportion of patients who showed an overall improvement $(\mathrm{CGI}-\mathrm{C}<4)$ at Week 12, versus placebo-treated patients (LOCF: $39.1 \%$ vs. $28.2 \%, p=0.033$; OC: $39.1 \%$ vs. $28.6 \%, p=0.031$ ) (Fig. 3; Table 2). At Week 12 , memantine treatment was not associated with significantly higher response rates than placebo on CGI Social Interaction or CGI Communication, although a significant effect of memantine was seen at Week 8 on both measures (Social Interaction, LOCF: $p=0.041$; OC: $p=0.036$; Communication, LOCF: $p=0.006$; OC: $p=0.005)$. Similar to the ASHA FACS subscales of SC and $\mathrm{CBN}$, the ASHA-FACS total score also demonstrated that caregivers of memantine-treated patients experienced significantly better patient communication than caregivers of placebo-treated patients at Week 12 (LOCF: $p=0.010$; OC: $p=0.013$ ). No significant difference between groups was observed on the OPT at Week 12 (LOCF: $p=0.185$; OC: $p=0.099$ )

\section{Safety and tolerability}

Four $(3.1 \%)$ placebo-treated patients and three (2.2\%) patients treated with memantine discontinued the trial due to an AE (Fig. 1). Overall TEAEs are summarized in Table 3. The TEAE profile was similar between the two groups; dizziness, restlessness, and headache were the only TEAEs reported by $\geq 2.0 \%$ patients, and that were higher in the memantine group (Table 3). A total of $13(10.1 \%)$ patients in the placebo group and four $(3.0 \%)$ patients in the memantine group experienced an SAE, none of which was judged related to the study drug. No patients in the memantine group died during the trial; one $(0.8 \%)$ patient in the placebo group died. Potentially clinically significant (PCS) adverse events that occurred in more memantine-treated patients than placebo-treated patients were a weight increase of $\geq 7 \%$ (2.3\% vs. $0 \%)$, a weight decrease of $\geq 7 \%(2.3 \%$ vs. $0 \%)$, and a diastolic blood pressure measurement of $\geq 180 \mathrm{~mm} \mathrm{Hg}$ that also represented a change of at least $20 \mathrm{~mm} \mathrm{Hg}$ over Baseline (1.5\% vs. 0\%).

\section{DISCUSSION}

This study, to our knowledge, is the first published prospective, double-blind, placebo-controlled study examining the benefits of an anti-dementia agent on communication abilities in patients with AD. Memantine treatment was found to be numerically superior to placebo for the primary efficacy parameter of this study, the FLCI score change at Week 12, but the treatment difference failed to reach statistical significance. Caregivers of memantine-treated patients reported significantly improved patient communication (OC and LOCF) compared with caregivers of 

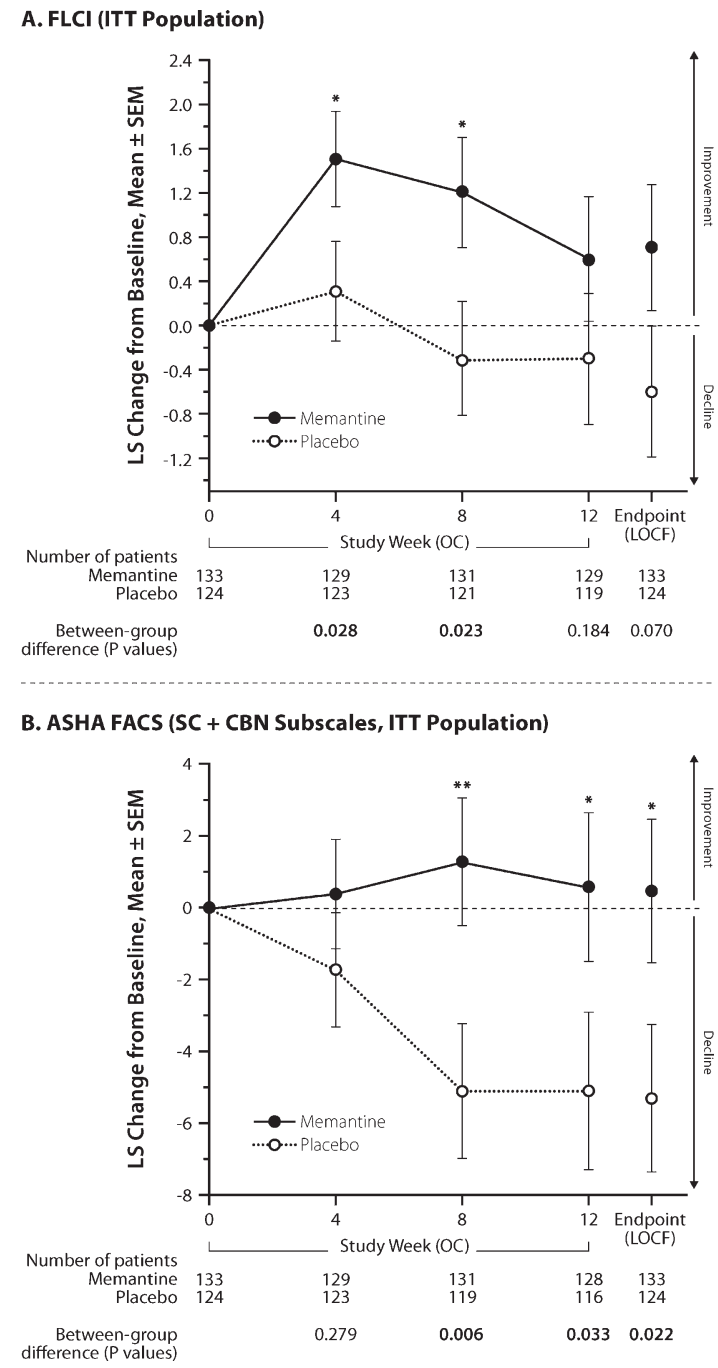

Fig. 2. A) Compared with placebo, memantine did not significantly improve functional communication at study Endpoint, as assessed using the FLCI, although the effect was significantly in favor of memantine at Weeks 4 and 8. B) Memantine treatment significantly improved functional communication compared with placebo at study Endpoint and Weeks 8 and 12, based on an assessment of caregivers, using the CBN and SC subscales of the ASHA FACS. ASHA FACS indicates the American Speech-Language-Hearing Association Functional Assessment of Communication Skills for Adults scale; CBN, Communication of Basic Needs subscale; FLCI, Functional Linguistics Communication Inventory; ITT, Intent-to-Treat; LOCF, last observation carried forward; LS, least squares; MMRM, mixed-effects model with repeated measures; OC, observed cases; SC, Social Communication subscale; SEM, standard error of the mean. ${ }^{*} p<0.05$; ${ }^{*} p<<0.01$; bold $\mathrm{P}$ values indicate those attaining statistical significance.

placebo-treated patients on the ASHA FACS subscales, from Week 8 onward (Fig. 2). A significant difference was also observed between the groups at Week 12 on the global outcome measure, and a higher percent- age of patients treated with memantine improved when compared with patients receiving placebo (Fig. 3). In addition, memantine treatment was associated with a low incidence of adverse events (only dizziness and restlessness were notably higher in the memantinetreated group), suggesting that memantine use in this patient population was not limited by adverse events.

These results are consistent with other prospective and post hoc analyses of communication in patients with AD treated with memantine. Schmitt et al. [11] reported that memantine was associated with significantly better performance than placebo on the language domain of the SIB in a trial of patients with moderate to severe AD concurrently taking a ChEI [26], and several other post hoc analyses have demonstrated evidence of memantine-treated patients significantly outperforming placebo-treated patients on a variety of languageand communication-based subscales $[10,28]$. Similarly, significant benefits of memantine versus placebo were seen on the prospectively defined measure of verbal fluency in patients with moderate to severe $\mathrm{AD}$ from a randomized, double-blind, placebo-controlled trial of extended-release memantine [13]. Post hoc analyses of the ChEIs donepezil [29, 30] and rivastigmine [9] have also demonstrated a superiority of drug treatment over placebo on domains of language in patients with $\mathrm{AD}$. Interestingly, a post-hoc analysis of our data revealed no significant treatment-by-AChEIusage interaction on the FLCI ( $p=0.9497$; LOCF) or ASHA FACS ( $p=0.1335$; LOCF) at study Endpoint across both groups.

Although several post hoc analyses of language and communication abilities have been performed on data obtained in antidementia drug trials $[9,11,13$, $28,29]$, to our knowledge this study is unique in that it is the only prospective, randomized, doubleblind, placebo-controlled trial to utilize a language and communication instrument as the primary outcome measure. The choice of one patient-based (FLCI) and one caregiver-based (ASHA FACS) outcome measure offered complementary insights into patients' communication abilities. Both the FLCI [2, 17] and the ASHA FACS $[5,22]$ have been validated for use in patients with AD, and ASHA FACS scores (Portuguese version) have been shown to correlate significantly with ADAS-cog scores in both individuals with AD $(r=-0.69, p<0.001)$ and in age-matched controls $(r=-0.63, p<0.001)$ [5]. A recent open-label trial of memantine, which was designed in part to complement this study, also investigated FLCI performance in patients with moderate to severe $\mathrm{AD}$ [16]. In that trial, once-daily $(20 \mathrm{mg})$ memantine treatment was associ- 
Table 2

Summary of efficacy (week 12, ITT population, LOCF)

\begin{tabular}{|c|c|c|c|c|c|c|}
\hline \multirow[t]{2}{*}{ Parameter } & \multicolumn{2}{|c|}{ Baseline score* $^{*}$} & \multicolumn{4}{|c|}{ Week 12 (Endpoint) } \\
\hline & $\begin{array}{l}\text { Placebo } \\
(n=124)\end{array}$ & $\begin{array}{c}\text { Memantine } \\
(n=133)\end{array}$ & $\begin{array}{l}\text { Placebo }^{\dagger} \\
(n=124)\end{array}$ & $\begin{array}{c}\text { Memantine }^{\dagger} \\
(n=133)\end{array}$ & $\begin{array}{c}\text { LSMD } \\
{[95 \% \mathrm{CI}]}\end{array}$ & $p$ value \\
\hline FLCI & $67.8 \pm 11.3$ & $68.7 \pm 11.1$ & $-0.6 \pm 0.6$ & $0.7 \pm 0.6$ & $1.3[-0.1,2.8]$ & $0.070^{\ddagger}$ \\
\hline \multirow[t]{2}{*}{ ASHA FACS $(\mathrm{SC}+\mathrm{CBN})$} & $137.8 \pm 28.1$ & $134.7 \pm 30.2$ & $-5.3 \pm 2.1$ & $0.5 \pm 2.0$ & $5.9[0.9,10.9]$ & $0.022^{\ddagger}$ \\
\hline & \multicolumn{6}{|c|}{ Participants, $n(\%)$} \\
\hline \multicolumn{7}{|l|}{$\overline{\text { CGI-C }}$} \\
\hline Improvement (Scores 1-3) & NA & NA & $35(28.2)$ & $52(39.1)$ & NA & \\
\hline No Change (Score 4) & NA & NA & $43(34.7)$ & $45(33.8)$ & NA & $0.033 \S$ \\
\hline Worsening (Scores 5-7) & NA & NA & $46(37.1)$ & $36(27.1)$ & NA & \\
\hline
\end{tabular}

${ }^{*}$ Mean \pm SD. ${ }^{\dagger}$ Least Squares Mean Difference \pm SEM. ${ }^{\ddagger}$ Based on Analysis of Covariance (ANCOVA; see Materials and Methods); ${ }^{P}$ value for the 7-category outcome measure; based on a Cochran-Mantel-Haenszel test (see Materials and Methods). CGI-C is a measure of change from Baseline; therefore, Baseline values are not applicable. ASHA FACS indicates the American Speech-Language-Hearing Association - Functional Assessment of Communication Skills for Adults scale; CBN, Communication of Basic Needs; CGI-C, clinical global impressions of change; CI, confidence interval; FLCI, Functional Linguistic Communication Inventory; ITT, Intent-to-Treat; LOCF, last observation carried forward; LSMD, least squares mean difference; NA, not applicable; SC, Social Communication; SD, standard deviation; SEM, standard error of the mean.

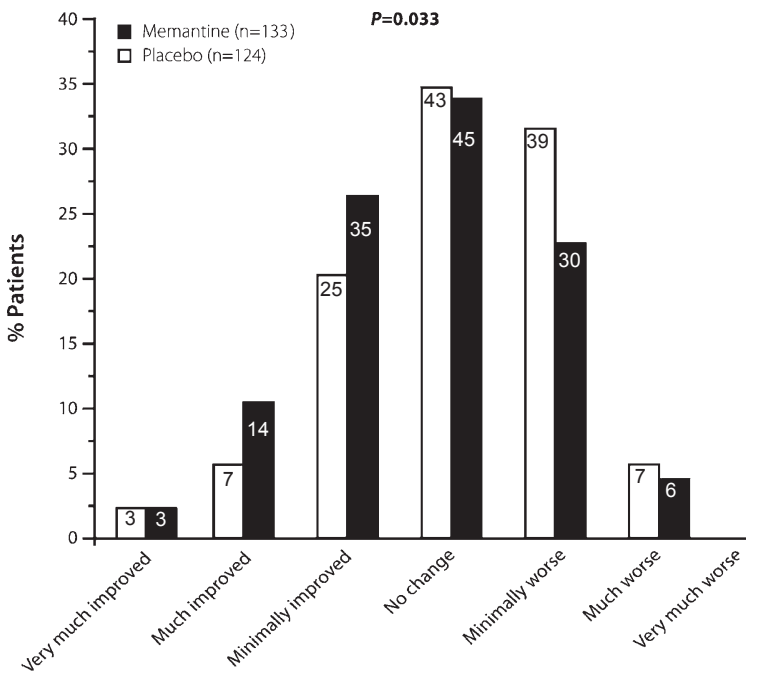

Fig. 3. Clinical Global Impression of Change at Week 12 (ITT Population; LOCF).

Patients treated with memantine performed significantly better on the CGI-C than those treated with placebo. Numbers inside the bars indicate numbers of patients that were scored to each category. $p$ values were obtained using a Cochran-Mantel-Haenszel test. ITT indicates Intent-to-Treat; LOCF, last observation carried forward.

ated with significant improvement on the FLCI after 12 weeks [16], an effect that persisted after a 4-week washout period. It should be noted, however, that the open-label study cannot be directly compared with our study since it was unblinded and did not include a placebo comparator.

The outcome of our trial is consistent with the communication-related measures from other trials in $\mathrm{AD}$; however, the interpretation of our results may be
Table 3

Summary of treatment-emergent adverse events (safety population)*

\begin{tabular}{lcc}
\hline TEAE & $\begin{array}{c}\text { Placebo } \\
(n=129)\end{array}$ & $\begin{array}{c}\text { Memantine } \\
(n=135)\end{array}$ \\
\hline Any TEAE & $64(49.6)$ & $66(48.9)$ \\
Dizziness & $2(1.6)$ & $7(5.2)$ \\
Upper respiratory tract infection & $5(3.9)$ & $4(3.0)$ \\
Fall & $4(3.1)$ & $4(3.0)$ \\
Hypertension & $4(3.1)$ & $4(3.0)$ \\
Edema, peripheral & $4(3.1)$ & $3(2.2)$ \\
Headache & $2(1.6)$ & $3(2.2)$ \\
Restlessness & $0(0.0)$ & $3(2.2)$ \\
Diarrhea & $5(3.9)$ & $2(1.5)$ \\
Nausea & $3(2.3)$ & $2(1.5)$ \\
Agitation & $4(3.1)$ & $0(0.0)$ \\
Syncope & $3(2.3)$ & $0(0.0)$ \\
\hline
\end{tabular}

*Data $[n(\%)]$ include all treatment-emergent adverse events (TEAEs) experienced by at least $2.0 \%$ of patients in either treatment group.

limited due to the modest sample size, a short duration of treatment (12 weeks), and the exploratory use of 2 instruments not commonly utilized in AD trials. In addition, the clinical relevance of a treatment difference (LSMD) of 1.3 points on the FLCI, and of 5.9 points on the ASHA FACS in a population of patients with $\mathrm{AD}$ is unknown.

\section{CONCLUSIONS}

In patients with moderate $\mathrm{AD}$, memantine is a welltolerated treatment option that may be associated with improvements in functional communication skills, particularly as recognized by caregivers. In addition, the FLCI and ASHA FACS, used here for the first time 
in a randomized clinical trial of $\mathrm{AD}$, may be useful tools for assessing communication-related declines and treatment benefits in patients with AD.

\section{ACKNOWLEDGMENTS}

The authors gratefully acknowledge Drs. Kathryn Bayles and Audrey Holland for providing consultation and guidance regarding the efficacy measures used in this study, Dr. E. Malca Resnick (formerly of Forest Research Institute; FRI) for study conceptualization and conduct, Sung Yun Yu from FRI for assistance in conducting the study, Dr. Yiqun $\mathrm{Hu}$ (formerly of FRI) for serving as the medical monitor for this study, Quintiles for providing clinical trial services and Dr. Michael Tocco from FRI and TorreyBeth Volkman from Prescott Medical Communications Group for editorial assistance.

Forest Laboratories, Inc. sponsored this trial and provided financial, material, and statistical support.

Authors' disclosures available online (http://www.jalz.com/disclosures/view.php?id=977).

The Memantine Study Group consisted of the following members: D. Ames, University of Melbourne Academic Unit for Psychiatry of Old Age, St. George's Hospital, Kew, Victoria, Australia; F. Badenhorst, Panorama Medi-Clinic, Cape Town, SA; K. Boundy, The Queen Elizabeth Hospital, Woodville South, Australia; J. Breedt, Pretoria, SA; H. Brodaty, Prince of Wales Hospital, Randwick, Australia; A. Brodtmann, Eastern Melbourne Neurosciences, Box Hill, Australia; R. Clarnette, The McCusker Foundation for Alzheimers' Disease Research, Perth, Australia; D. Crimmins, Central Coast Neuroscience Research, East Gosford, Australia; C. Davis, The Prince Charles Hospital, Chermside, Australia; M. Gani, GCT Trial Centre, Port Elizabeth, SA; J. Green, St. Augustine's Hospital, Durban, SA; M. Hills, Timaru Hospital; Timaru, New Zealand; M. Isaacs, Medical \& Dental Centre, Rosebank, SA; S. Kurrle, Hornsby Ku-RingGai Hospital, Hornsby, Australia; S. Lipschitz, The Memory Center, Johannesburg, SA; S. Macfarlane, Frankston Hospital, Frankston, Australia; R. Prowse, Royal Adelaide Hospital, Adelaide, South Australia; P. Schofield, James Fletcher Hospital, Newcastle, Australia; R. Schwartz, Southern Neurology, Kogarah, Australia; E. Tan, Toowoomba Base Hospital, Toowoomba, Australia; J. Thorne, Excellentis Clinical Trial Consultants, George, SA; P. Wood, The Memory Clinic, North Shore, New Zealand.

\section{REFERENCES}

[1] Honig LS, Mayeux R (2001) Natural history of Alzheimer's disease. Aging (Milano) 13, 171-182.

[2] Bayles KA, Tomoeda CK, Cruz RF, Mahendra N (2000) Communication abilities of individuals with late-stage Alzheimer disease. Alzheimer Dis Assoc Disord 14, 176-181.

[3] Forstl H, Kurz A (1999) Clinical features of Alzheimer's disease. Eur Arch Psychiatry Clin Neurosci 249, 288-290.

[4] Fromm D, Holland A (1989) Functional communication in Alzheimer's disease. J Speech Hear Disord 54, 535-540.

[5] de Carvalho IA, Mansur LL (2008) Validation of ASHA FACS-functional assessment of communication skills for Alzheimer disease population. Alzheimer Dis Assoc Disord 22, 375-381.

[6] American Speech-Language-Hearing Association. (2006) Preferred Practice Patterns for the Profession of Audiology [Preferred Practice Patterns]. Available from http://www. asha.org/policy

[7] Ripich DN (1994) Functional communication with AD patients: a caregiver training program. Alzheimer Dis Assoc Disord 8(Suppl 3), 95-109.

[8] Wilcox T, Chen W, O' Quinn S, Setyawan J, Saxton J, Erder M (2010) Effectiveness of memantine in patients with moderate to severe Alzheimer's disease, as measured by the Caregiver Perceived Burden Questionnaire (CPBQ) [abstract]. Neurology 74, A270.

[9] Farlow MR, Cummings JL, Olin JT, Meng X (2010) Effects of oral rivastigmine on cognitive domains in mild-to-moderate Alzheimer's disease. Am J Alzheimers Dis Other Demen 25, 347-352.

[10] Ferris S, Ihl R, Robert P, Winblad B, Gatz G, Tennigkeit F, Gauthier S (2009) Treatment effects of Memantine on language in moderate to severe Alzheimer's disease patients. Alzheimers Dement 5, 369-374.

[11] Schmitt FA, van Dyck CH, Wichems CH, Olin JT (2006) Cognitive response to memantine in moderate to severe Alzheimer disease patients already receiving donepezil: an exploratory reanalysis. Alzheimer Dis Assoc Disord 20, 255-262.

[12] Saxton J, Tocco M, Hofbauer RK, Graham SM (2009) Effects of memantine on language and functional communication in patients with moderate to severe Alzheimer's disease: a pooled analysis [abstract]. J Am Geriatr Soc 57, S170.

[13] Grossberg G, Manes F, Allegri R, Robledo LMG, Gloger S, Xie L, D JX, Perhach JL, Graham SM (2008) A multinational, randomized, double-blind, placebo-controlled, parallel-group trial of memantine extended-release capsule (28mg, once daily) in patients with moderate to severe Alzheimer's disease. Alzheimers Dement 4, T793.

[14] Berthier ML, Green C, Higueras C, Fernandez I, Hinojosa J, Martin MC (2006) A randomized, placebo-controlled study of donepezil in poststroke aphasia. Neurology 67, 1687-1689.

[15] Berthier ML, Green C, Lara JP, Higueras C, Barbancho MA, Davila G, Pulvermuller F (2009) Memantine and constraintinduced aphasia therapy in chronic poststroke aphasia. Ann Neurol 65, 577-585.

[16] Schulz JB, Rainer M, Klunemann HH, Kurz A, Wolf S, Sternberg K, Tennigkeit F (2011) Sustained effects of oncedaily memantine treatment on cognition and functional communication skills in patients with moderate to severe Alzheimer's disease: results of a 16-week open-label trial. J Alzheimers Dis 25, 463-475.

[17] Bayles KA, Tomoeda CK (1994) Functional Linguistic Communication Inventory Canyonlands Publishing Co., Tucson, AZ. 
[18] Frattali CM, Thompson CK, Holland AL, Wohl CB, Ferketic MM (1995) Functional Assessment of Communication Skills for Adults (ASHA FACS) American Speech-LanguageHearing Association, Rockville, MD

[19] Folstein MF, Folstein SE, McHugh PR (1975) "Mini-mental state". A practical method for grading the cognitive state of patients for the clinician. J Psychiatr Res 12, 189-198.

[20] Rosen WG, Terry RD, Fuld PA, Katzman R, Peck A (1980) Pathological verification of ischemic score in differentiation of dementias. Ann Neurol 7, 486-488.

[21] Bayles KA, Tomoeda CK (1993) Arizona Battery for Communication Disorders of Dementia Canyonlands Publishing Co., Tucson, AZ.

[22] Paul D, Frattali C, Holland A (2004) Functional Assessment of Communication Skills for Adults (ASHA FACS): Addendum American Speech-Language-Hearing Association, Rockville, $\mathrm{MD}$

[23] Reisberg B, Schneider L, Doody R, Anand R, Feldman H, Haraguchi H, Kumar R, Lucca U, Mangone CA, Mohr E, Morris JC, Rogers S, Sawada T (1997) Clinical global measures of dementia. Position paper from the International working group on harmonization of dementia drug guidelines. Alzheimer Dis Assoc Disord 11(Suppl 3), 8-18.

[24] Stern R, White T (2003) Neurological Assessment Battery Psychological Assessment Resources, Inc., Lutz, FL.
[25] Reisberg B, Doody R, Stoffler A, Schmitt F, Ferris S, Mobius HJ (2003) Memantine in moderate-to-severe Alzheimer's disease. $N$ Engl J Med 348, 1333-1341.

[26] Tariot PN, Farlow MR, Grossberg GT, Graham SM, McDonald S, Gergel I (2004) Memantine treatment in patients with moderate to severe Alzheimer disease already receiving donepezil: A randomized controlled trial. JAMA 291, $317-$ 324.

[27] van Dyck CH, Tariot PN, Meyers B, Resnick EM (2007) A 24week randomized, controlled trial of memantine in patients with moderate-to-severe Alzheimer disease. Alzheimer Dis Assoc Disord 21, 136-143.

[28] Tocco M, Graham SM (2010) Effects of memantine treatment on language abilities and functional communication in patients with moderate-to-severe Alzheimer's disease: A review of data. Alzheimers Dement 6, S304-

[29] Lopez-Pousa S, Vilalta-Franch J, Garre Olmo J, TuronEstrada A, Lozano-Gallego M, Hernandez-Ferrandiz M, Fajardo-Tibau C, Cruz-Reina MM (2001) Effectiveness of donepezil on several cognitive functions in patients with Alzheimer's disease over 12 months. Neurologia 16, 342-347.

[30] Schwam E, Xu Y (2010) Cognition and Function in Alzheimer's Disease: Identifying the Transitions from Moderate to Severe Disease. Dement Geriatr Cogn Disord 29, 309-316. 